\title{
Concertation sociale et négociation collective au Québec en temps de crise
}

Restructurations et découplage

Social dialogue and collective bargaining in Quebec during the economic crisis:

restructuring and decoupling

\section{Mélanie Laroche et Philippe Barré}

\section{OpenEdition}

\section{Journals}

Édition électronique

URL : http://journals.openedition.org/travailemploi/5854

DOI : 10.4000/travailemploi.5854

ISSN : 1775-416X

\section{Éditeur}

DARES - Ministère du Travail

\section{Édition imprimée}

Date de publication : 15 décembre 2012

Pagination : 65-77

ISSN : 0224-4365

\section{Référence électronique}

Mélanie Laroche et Philippe Barré, «Concertation sociale et négociation collective au Québec en

temps de crise », Travail et Emploi [En ligne], 132 | octobre-décembre 2012, mis en ligne le 01 décembre 2014, consulté le 01 mai 2019. URL : http://journals.openedition.org/travailemploi/5854 ; DOI :

10.4000/travailemploi.5854 


\title{
Concertation sociale et négociation collective au Québec en temps de crise : restructurations et découplage
}

\author{
Mélanie Laroche ${ }^{(*)}$, Philippe Barré(**)
}

Le Québec semble avoir échappé aux effets de la crise économique et financière de 2008-2009 en maintenant inchangé son niveau d'emploi. Cet article analyse en profondeur le rôle qu'ont joué à cet égard les acteurs syndicaux et patronaux au sein des institutions de concertation sociale de cette province. Ces dernières constituent une exception à l'échelle nord-américaine et rapprochent le modèle québécois de relations industrielles de certains systèmes européens. Les mesures d'emploi qu'ils ont utilisées ou mises en place ont contribué à éviter bon nombre de licenciements collectifs. Le maintien de l'emploi durant cette période doit aussi être interprété à la lumière d'une transformation majeure de la structure économique du Québec dans laquelle des pertes massives d'emplois dans les secteurs manufacturiers ont été compensées par des créations d'emplois dans les secteurs des services. L'analyse du contenu de plus de six mille conventions collectives conclues au Québec, sur une période de quinze ans, est cependant révélatrice d'un découplage entre la concertation sociale qui s'opère à des niveaux centralisés (provincial, sectoriel, régional) et la négociation collective décentralisée au niveau de l'établissement. La mise en évidence de ce décalage est révélatrice d'enjeux importants liés à la capacité des acteurs syndicaux et patronaux du Québec de participer à la modernisation de l'économie de cette province.

La dernière crise économique et financière a pris la forme d'une récession mondiale qui a touché l'ensemble des économies, y compris les secteurs industriels et de services à haute valeur ajoutée. Les conséquences sur les conditions d'emploi et de travail ont été importantes : licenciements collectifs, mises à pied temporaires, gel des salaires, coupure dans les avantages sociaux, pertes importantes dans les fonds de retraite privés et publics, etc. En Europe, il semble que les pays qui possèdent les systèmes les plus développés et les plus institutionnalisés de dialogue social sont ceux qui ont réagi le plus rapidement à la crise en favorisant notamment la tenue de sommets tripartites nationaux (entre l'État, les syndicats de salariés et les organisations patronales), la création d'organisations d'aide au reclassement, des mesures actives d'emplois, etc. (Eurofound, 2009). Qu'en est-il de la situation du Canada, et plus spécifiquement de la province du Québec? Quels ont été les effets de la crise sur l'économie et l'emploi? Comment les organisations syndicales et patronales ont-elles réagi à ce choc important?

\footnotetext{
(*) École de relations industrielles, Université de Montréal, Centre de recherche interuniversitaire sur la mondialisation et le travail; melanie.laroche.2@umontreal.ca

$(* *)$ École de relations industrielles, Université de Montréal, Centre de recherche interuniversitaire sur la mondialisation et le travail; philippe.barre@umontreal.ca
}

Le Québec offre un terrain d'analyse riche car il est atypique à l'échelle de l'Amérique du Nord. Le système de rapports collectifs qui a été instauré dans cette province canadienne a historiquement favorisé le développement d'un dialogue social axé sur des relations bipartites institutionnalisées. La négociation collective a ainsi permis aux acteurs patronaux et syndicaux de participer démocratiquement à l'élaboration des règles du travail. En marge de ce système de négociation collective décentralisée au niveau de l'établissement (typique des systèmes nord-américains de relations collectives de travail), s'est aussi déployée très tôt dans le paysage institutionnel québécois une tradition de concertation supra-entreprise. Déjà dans les années 1930, la Loi relative à l'extension des conventions collectives, à l'origine d'un mécanisme d'extension des accords négociés à des parties non signataires, a favorisé l'implantation de comités paritaires au sein de plusieurs secteurs économiques au Québec où les partenaires ont eu à échanger sur des questions en lien avec la croissance industrielle. Dans la mouvance de la «Révolution tranquille»(1), cette tendance s'est affirmée plus nettement et

(1) La Révolution tranquille est la période couvrant la décennie 1960 au cours de laquelle de nombreuses réformes sociales ont été lancées. L'État québécois a alors adopté les principes de l'Etat-providence et l'identité nationale québécoise s'est forgée (Godin, 1991). 
plusieurs innovations institutionnelles ont été mises en place afin que les partenaires sociaux puissent échanger sur divers enjeux. Au fil des années, plusieurs lieux d'échanges entre partenaires sociaux ont été créés pour agir à différents niveaux, autant provincial, que sectoriel, régional ou local. Les relations du travail au Québec s'inscrivent donc au sein d'un modèle hybride, associant la négociation collective et la concertation sociale, permettant ainsi aux acteurs d'agir au-delà des frontières de l'entreprise.

Nous faisons l'hypothèse dans cet article que la concertation sociale a influencé favorablement le maintien de l'emploi au cours de la crise économique et financière de 2008-2009. À la différence de la majorité des pays européens, des États-Unis, et même des autres provinces canadiennes, le Québec n'a en effet connu en 2009 qu'une très légère baisse de son niveau d'emploi, malgré un recul de son PIB (produit intérieur brut) équivalent à celui observé ailleurs au Canada et dans les autres pays industrialisés.

Notre analyse vise à identifier le rôle et la portée des mécanismes de concertation sociale qui ont été mobilisés au Québec pour faire face à la crise économique et financière de 2008 et 2009. Après une présentation de la méthodologie de recherche, nous mettons en évidence la situation exceptionnelle du Québec sur le plan de l'emplois lors de cette crise. Derrière une relative solidité de l'économie québécoise et des pertes d'emplois apparemment limitées, se cache cependant une restructuration majeure du marché du travail et de l'organisation industrielle de cette province. Les mesures de préservation de l'emploi qui ont été mises en place par les partenaires sociaux du Québec au sein de certaines institutions de concertation sociale ne semblent avoir été efficaces qu'au sein d'une partie seulement du secteur privé. Les entreprises à plus faible intensité technologique, en particulier dans le secteur manufacturier, ont continué à licencier leurs personnels dans le cadre de réductions partielles ou totales de leurs activités. Ces mesures n'ont donc pas permis d'inverser, ou de ralentir, les pertes massives d'emplois observées depuis le milieu des années 2000 dans certains secteurs industriels. Nous examinons ensuite les mesures d'urgence élaborées par les partenaires sociaux au sein des institutions de concertation sociale afin d'amoindrir les chocs causés par cette crise. Leur analyse permet de porter un regard critique sur la portée réelle de la concertation au Québec. Elle met en évidence les difficultés structurelles des mécanismes de concertation sociale à se coupler au système décentralisé de négociation collective au niveau de l'établissement. Les mesures décidées aux niveaux provinciaux et sectoriels de concertation semblent en effet trouver difficilement écho dans la nature et le contenu des conventions collectives négociées dans les établissements. Dans le contexte de crise économique et financière marquée par une très forte pression sur l'emploi, nos données indiquent que les acteurs qui agissent au niveau de l'entreprise adoptent des stratégies essentiellement défensives qui ne tiennent pas compte des stratégies de modernisation de l'économie et du marché du travail prônées par les acteurs impliqués dans les institutions de concertation sociale.

\section{Méthodologie de recherche}

Cette recherche s'inscrit dans le cadre d'un programme lancé en 2010 en vue de faire le point sur le dialogue social au Québec. Nous avons alors rencontré près d'une quinzaine de personnes agissant pour le compte des grandes confédérations et centrales syndicales, des fonds d'investissement de travailleurs, des associations patronales et des institutions publiques et paritaires du marché du travail. Ces entretiens ont abordé quatre grandes thématiques, à savoir la nature des enjeux et les objectifs poursuivis par les instances de dialogue social au Québec, les niveaux variés de régulation, les difficultés auxquelles les acteurs engagés dans ces lieux de concertation sont confrontés et les voies de modernisation des lois du travail pour faciliter le dialogue social au Québec.

Suite à cette première phase de la recherche, nous avons ciblé quatre axes principaux sur lesquels un consensus a été établi entre les partenaires sociaux au regard de la trajectoire à privilégier afin de favoriser la compétitivité des entreprises, plus particulièrement leur productivité, et la qualité de vie des travailleurs québécois. Nous aborderons, dans l'ordre, l'implication des travailleurs et de leur syndicat dans les décisions, une meilleure protection des travailleurs, la répartition équitable et transparente des gains de productivité, et l'investissement dans la main-d'œuvre, par exemple par la formation ou l'amélioration des compétences (MESS, 2005). Ces axes prioritaires ont été définis par une équipe de travail multipartite, les Partenaires pour la compétitivité et l'innovation sociale, formée par des représentants des grandes centrales syndicales québécoises, des associations patronales et du gouvernement du Québec. Ce groupe était particulièrement préoccupé par les moyens à mettre en œuvre pour renforcer le dialogue social au Québec, lequel est considéré comme un élément clé du processus de développement équilibré et harmonieux de l'économie québécoise.

Afin de mesurer l'existence d'un éventuel couplage entre les niveaux où s'exercent la concertation sociale et la négociation collective dans l'établissement, nous avons étudié les transformations du contenu des conventions collectives, lesquelles sont le reflet des compromis entre les intérêts promus par les parties. En nous basant sur 
les données du ministère du Travail du Québec(2), nous avons analysé l'évolution des dispositions conventionnelles entre deux périodes de référence (1988-1991 et 2006-2009), sur plus de quinze ans ${ }^{(3)}$. Cette étude avait pour principal but d'évaluer dans quelle mesure la tradition de concertation s'est aussi développée à l'échelle de l'entreprise. Nous visions également à vérifier l'adéquation des ententes conclues à l'échelle des entreprises avec les quatre axes de développement du dialogue social établis par les partenaires sociaux.

Pour ce faire, nous avons ciblé cinq types de dispositions conventionnelles : les clauses en lien avec la participation à la gestion (comités paritaires traitant d'enjeux divers), la protection des emplois, les salaires ${ }^{(4)}$, la transparence ou la transmission de l'information économique et la formation. Précisons que ces dispositions reprennent également les principales caractéristiques des anciens contrats sociaux ${ }^{(5)}$, et, plus largement, les ententes de longue durée (Mict, 1993 ; BERgeron, Bourque, 1996). Or, cette volonté de conclure de tels contrats constitue pour certains auteurs un signe manifeste de la mutation des relations entre les parties patronales et syndicales (Morin, 1993) autrement dit,

(2) Les données sont tirées des publications du ministère du Travail à chacune des époques ciblées par la recherche : Ministère du Travail, Conditions contenues dans les conventions collectives, Québec, Publications du Québec, diverses années, et Portrait statistique des conventions collectives analysées au Québec, Québec, Direction de l'information sur le travail, diverses années.

(3) $\mathrm{La}$ raison première de recourir spécifiquement à ces périodes est la disponibilité des données, l'analyse des conventions collectives de 1992 à 2002 n'ayant pas fait l'objet de publication. En revanche, l'intérêt d'examiner des périodes relativement éloignées dans le temps nous a paru évident afin de dégager des tendances de fond plutôt que des orientations conjoncturelles. De plus, le contexte a beaucoup évolué entre les deux périodes. Par ailleurs, il était important de faire une comparaison par séquence de quatre ans pour aller au-delà des variations annuelles dans les conventions signées, qui dépendent souvent davantage de la composition du groupe de conventions collectives analysées que de l'existence de réelles tendances. Cela nous permettait d'obtenir un groupe le plus large possible de conventions dont la durée moyenne se situe aux alentours de quatre ans. Mentionnons que, pour certaines clauses, cette comparaison entre les périodes 1988-1991 et 2006-2009 n'a pu être effectuée, les données n'étant pas disponibles pour l'une ou l'autre de ces périodes.

(4) Les données salariales sont tirées de : Ministère du Travail du Québec, Indice de croissance des taux de salaires négociés : bilans, Québec, Publications du Québec, diverses années. Les données sur l'inflation qui ont servi au calcul des augmentations du salaire réel proviennent du site Internet de l'Institut de la statistique du Québec (http://www.stat.gouv.qc.ca/princ_indic/ default.htm\#tab).

(5) Le ministère de l'Industrie, du Commerce et de la Technologie (MICT) a identifié sept éléments caractéristiques des contrats sociaux : la transparence économique et de gestion, la démarche de qualité totale, l'élaboration et l'application d'un plan de développement des ressources humaines, la stabilité de l'emploi, la capacité d'adaptation par la flexibilité et la mobilité dans l'organisation du travail, l'élaboration d'un mécanisme de gestion de l'entente et les dispositions garantissant la poursuite des opérations de l'entreprise au-delà de la durée convenue dans la convention collective. du passage d'un mode antagoniste à des relations plus harmonieuses et continues entre les acteurs. Les dispositions conventionnelles ciblées sont ainsi pertinentes pour évaluer la qualité du dialogue social au sein des entreprises.

\section{Les effets de la crise économique et financière sur l'emploi}

Le Québec, à l'instar des autres économies avancées, a été touché par la crise économique et financière de 2008-2009. Son économie a connu une période de récession qui a débuté à partir du quatrième trimestre 2008 et qui s'est terminée à la fin du deuxième trimestre 2009. Son PIB a diminué de $2,1 \%$ en 2009 , ce qui est comparable au recul de l'ensemble de l'économie canadienne (- 2,5\%), l'économie américaine (- $2,63 \%)$, ou certains pays de l'Union européenne (- 2,6\% en France, - $3 \%$ en Belgique), mais est très en deçà du recul du PIB de nombreux autres pays européens ${ }^{(6)}$.

\section{Des effets apparemment limités sur l'emploi}

$\mathrm{Au}$ Québec, cette récession n'a cependant eu qu'un impact relativement limité sur le nombre total d'emplois. 32000 emplois ont été perdus en 2009, ce qui représente un recul net de l'emploi de $0,8 \%$ (IsQ, 2011, p. 9). Ces destructions ont été nettement moins importantes que celles observées au cours des deux récessions précédentes. En 1982, l'emploi avait ainsi régressé au Québec de 5,4 \% (- 151100 emplois). En 1991 et 1992, ce sont respectivement $55900(-1,8 \%)$ et $45800(-1,5 \%)$ postes de travail qui avaient été perdus; le repli de l'emploi s'était alors poursuivi jusqu'en 1993 (-7700 emplois; $-0,3 \%)$.

Proportionnellement, les destructions d'emplois observées au Québec au cours de l'année 2009 ont été une fois et demie moins importantes que dans l'ensemble du Canada (- 1,6 \%; - 276900 emplois) et deux fois et demie moins importantes que dans les provinces de Terre-Neuve-et-Labrador (- 2,5\%; - 5400), de la Colombie-Britannique (-2,4\%; - 54900) et de l'Ontario $(-2,4 \% ;-161200)$ (IsQ, 2010, p. 35). Les pertes d'emploi du Québec sont sans commune mesure avec celles des États-Unis, qui ont vu reculer l'emploi de $3,8 \%$ au cours de l'année 2009 (OCDE, 2011). Elles sont également inférieures aux pertes de la plupart des États membres européens en 2009 (- 1,2 \% en France, - 1,6 \% en Italie, - 1,7 \% au Royaume-Uni, - 3,1\%

(6) L'ensemble de l'Union européenne a connu un recul de 4,2 \% de son PIB en 2009 (- 4,9\% en Allemagne, - 4,9\% au Danemark, - 7,1 \% en Irlande, - $4 \%$ aux Pays-Bas, - 4,9\% au Royaume-Uni, - $5 \%$ en Italie, - 14,8 \% en Lituanie, etc.) (Eurofound, 2010, p. 14). 
au Danemark, - 6,6 \% en Espagne, - 8,1 \% en Irlande, etc.); le Luxembourg, l'Allemagne et la Belgique font exception puisqu'ils ont connu respectivement une croissance $(+1 \%)$, une stabilité $(+0 \%)$ et une légère diminution $(-0,2 \%)$ de l'emploi en 2009 (Eurostat, 2011).

Plus du double des emplois détruits au Québec en 2009 ont cependant été récupérés au cours de l'année suivante. 66700 emplois $(+1,7 \%)$ ont ainsi été créés en 2010, ce qui porte le nombre total d'emplois au Québec à 3915100 et représente un sommet depuis 1976 (IsQ, 2011). Le PIB a quant à lui augmenté de 3,3\% en 2010 par rapport à l'année précédente. L'augmentation de l'emploi s'observe également dans la plupart des autres provinces du Canada, bien que dans des proportions plus limitées. Elle contraste cependant fortement avec la situation américaine dans laquelle l'emploi a continué à reculer en $2010(-0,8 \%)^{(7)}$ ainsi qu'en Europe où la grande majorité des États membres ont également connu de nouvelles pertes d'emploi au cours de 2010 : - 0,7 \% en Italie, - 0,7\% au Royaume-Uni, - 2,2 \% au Danemark, - 2,6 \% en Espagne, - 4,2 \% en Irlande, etc.; seuls la France $(+0,1 \%)$, l'Allemagne $(+0,5 \%)$, et la Belgique $(+0,8 \%)$ ont connu une légère croissance d'emploi ${ }^{(8)}$.

\section{Des conséquences différenciées selon les secteurs d'activité}

Comment expliquer l'impact relativement limité de la crise économique et financière et de la récession qu'elle a engendrée, sur l'emploi au Québec? Si l'on s'appuie sur les exemples de bon nombre de pays européens dans lesquels les partenaires sociaux et les gouvernements ont réagi rapidement à cette crise en mettant en place des mesures d'urgence de préservation de l'emploi et en concluant des accords bi- ou tripartites aux niveaux national ou sectoriel (FreysSINET, 2011; EUROFOUND, 2009 et 2010; Lefresne, Sauviat, 2011), doit-on attribuer aux institutions de concertation sociale québécoises la responsabilité d'avoir amorti ce choc économique?

Les résultats de notre enquête nous amènent à resituer l'influence qu'ont eue les dispositifs institutionnels de concertation sur le maintien de l'emploi à la lumière d'un mouvement plus large de restructuration industrielle. Le léger recul de l'économie en 2009, et sa croissance en 2010, masquent en effet une transformation profonde de l'économie du Québec. Des pans importants de celle-ci ont ainsi été atteints par la récession, avec des pertes massives d'emplois dans les secteurs manufacturiers, qui ont été compensées par des gains importants d'emplois dans les secteurs des services. Les secteurs des soins de santé $(+11300$ emplois), de l'aide sociale $(+17600)$ et des services

(7) OCDE (2011), OECD Employment Outlook.

(8) Eurostat (2011), Enquête sur les forces de travail. professionnels, scientifiques et techniques $(+8900)$ ont en effet connu en 2009 de très fortes augmentations d'emplois (IsQ, 2010). En 2010, les secteurs des services ont été à la base de plus de 83500 créations d'emplois (IsQ, 2011).

À l'inverse, la situation de l'industrie manufacturière s'est particulièrement détériorée. Alors que ce secteur était, jusqu'en 2009, le deuxième en importance en termes d'emplois à l'échelle de la province, il en a perdu 11400 en 2009, 32400 en 2010 et 13300 en 2011. Il est important de noter que la récession économique n'explique qu'en partie ces pertes qui s'inscrivent dans une tendance plus longue remontant au milieu des années 2000 (Bernard, 2009). Après une croissance de 1998 à 2000, l'emploi manufacturier du Québec a atteint un sommet en 2002 avec 650000 emplois $(19 \%$ de l'emploi total du Québec). Entre 2002 et 2010, ce secteur a cependant perdu le quart de ses emplois (-162500) et ne représente plus aujourd'hui que $13 \%$ de l'emploi total du Québec (IsQ, 2011).

Le maintien du niveau d'emploi total du Québec durant la récession de 2008-2009 masque ainsi une transformation très importante de la structure industrielle du Québec. Dans le secteur manufacturier, 1554 restructurations d'entreprises, impliquant le licenciement d'au moins dix travailleurs par entreprise, entre 2002 et 2008 ont été recensées (Jalette, Prudent, 2010). Elles ont pris la forme de fermetures définitives d'usines, de délocalisations, de fusions et d'acquisitions, de réduction partielle d'opérations, etc. De plus, $72 \%$ de ces restructurations touchent des entreprises à faible intensité technologique et se sont soldées par des fermetures d'usines ou des licenciements définitifs. Dans les industries à plus forte intensité technologique, comme les secteurs aéronautique ou pharmaceutique, elles ont eu pour conséquence des pertes d'emplois temporaires, liées à l'évolution de la demande ou à des événements d'ordre conjoncturel tandis que les industries à faible intensité technologique ont eu à faire face à une crise de nature structurelle. En résumé, les bons résultats du Québec en termes d'emplois s'expliquent, en partie du moins, par une transformation importante de l'économie en faveur des secteurs tertiaires.

\section{Rôle et portée des mécanismes de concertation sociale}

Le système de relations collectives de travail au Québec est typique du système nord-américain basé sur la négociation décentralisée. Il constitue cependant une exception à l'échelle de ce continent par les pratiques de concertation qu'il met en œuvre à des niveaux plus centralisés. Il constitue ainsi un modèle hybride entre le système nord-américain et certains systèmes européens de relations 
industrielles. Après avoir présenté la nature de ce modèle et le cadre institutionnel qui le constitue, nous examinons le rôle qu'ont joué les partenaires sociaux du Québec au sein des institutions de concertation sociale lors de la crise économique et financière de 2008-2009. Nous analysons les mesures d'urgence qu'ils ont prises et la portée de leur action dans la gestion de cette crise.

\section{Un système hybride}

Le système québécois de rapports collectifs est historiquement basé sur la négociation collective, décentralisée au niveau de l'établissement. Son régime juridique est directement issu du Wagner Act américain de 1935 (Piore, SABel, 1989, pp. 128-135), importé et transposé au Canada et au Québec, respectivement en 1943 et 1944. L'un de ses principes (Coutu et al., 2009, pp. 195-200) reconnaît le droit fondamental de tout salarié (à l'exception des cadres et des contremaîtres qui ne sont pas visés par ce régime) d'appartenir à une association de salariés de son choix. Cette association doit obligatoirement faire l'objet d'un processus d'accréditation de la part de l'État qui vérifie la validité des adhésions syndicales et s'assure que les conditions préalables à la négociation collective sont réunies. À la différence des systèmes européens de relations collectives de travail, le régime québécois repose sur l'existence d'un monopole syndical de représentation que l'accréditation confère en effet à l'association de salariés la plus représentative. Ce principe repose sur l'idée que la paix industrielle est plus facile à réaliser lorsqu'un seul syndicat est reconnu dans l'entreprise. Enfin, et de manière également spécifique aux systèmes nord-américains de relations industrielles, le modèle québécois de négociation collective est décentralisé au niveau de l'établissement ou de l'entreprise lorsque cette dernière ne comporte qu'un seul établissement.

Cependant, le Québec fait figure d'exception en Amérique du Nord par l'existence de plusieurs structures visant à mettre en œuvre une concertation sociale tripartite (syndicats, employeurs et gouvernement) ${ }^{(9)}$. Dans la mouvance des réformes des institutions publiques du Québec, plusieurs créations institutionnelles ont en effet permis de mettre en œuvre, aux niveaux provincial, sectoriel, régional et local trois modes de participation que sont les activités ponctuelles de concertation, les conseils d'administration d'organismes publics et les organismes consultatifs permanents.

Une première forme de participation institutionnelle regroupe donc les nombreuses activités informelles de consultation qui ont eu lieu, au fil de l'histoire, en marge par exemple des nombreux sommets socio-économiques (Pointe-au-Pic, 1977; Montebello, 1979; Québec, 1982), du Sommet sur l'économie et l'emploi de 1996, du Forum des générations de 2004 ou encore du Sommet de Lévis de 2010. Les partenaires sociaux ont ainsi eu l'occasion d'échanger, de manière ponctuelle, sur des questions relatives au développement économique et social du Québec. Bien que de tels exercices présentent une certaine utilité, ils ont souvent été critiqués par les acteurs sociaux, jugeant que leurs intérêts n'étaient pas toujours pris en compte (Rouillard, 2009).

Les représentants patronaux et syndicaux siègent en outre à de nombreux conseils d'administration d'organismes publics. Selon le degré d'implication de l'acteur syndical (GAGNON, 1991), nous distinguons deux groupes d'organismes : dans le premier, par exemple à la Caisse de dépôt et de placement du Québec, les syndicats occupent une minorité de sièges au conseil d'administration pour assurer un contrepoids à la présence d'administrateurs patronaux ou gouvernementaux; dans le deuxième, les acteurs syndicaux doivent nécessairement être présents au sein de leur conseil d'administration qui traitent d'enjeux directement liés aux marchés du travail. Nous retrouvons notamment dans cette catégorie la Commission de la santé et de la sécurité au travail (CSST), la Commission des normes du travail (CNT), la Commission des droits de la personne et l'Office de la langue française.

Enfin, les institutions permanentes de consultation (voir tableau 1) permettent essentiellement au Gouvernement du Québec de consulter les partenaires sociaux et de définir conjointement de nouvelles pratiques dans deux domaines principaux : le développement économique, d'une part, et la formation professionnelle et les relations de travail, d'autre part (ChARest, 2004, 2007). Au niveau provincial, les associations patronales et les centrales syndicales interviennent au sein du Conseil des partenaires économiques dans lequel les partenaires sociaux échangent et sont informés des grandes orientations du gouvernement en matière de développement économique. Ils siègent également au Conseil consultatif sur le travail et la maind'œuvre (CCTM) dont l'objectif est d'orienter et de soutenir l'action gouvernementale sur le travail et les relations professionnelles. Une autre institution enfin, la Commission des partenaires du marché du travail (CPMT), regroupe les représentants des employeurs, des syndicats, de l'enseignement, des organismes communautaires et des organismes gouvernementaux et a pour but d'améliorer le fonctionnement du marché du travail et de développer la qualification de la main-d'œuvre. 
Tableau 1 : Institutions de consultation et de concertation sociale au Québec

\begin{tabular}{|c|c|c|}
\hline \multirow{2}{*}{$\begin{array}{c}\text { Niveaux de } \\
\text { concertation }\end{array}$} & $\begin{array}{c}|c| \\
\text { Objectifs } \\
\text { économique }\end{array}$ & $\begin{array}{c}\text { Formation } \\
\text { professionnelle et } \\
\text { relations de travail }\end{array}$ \\
\hline Provincial & $\begin{array}{c}\text { Conseil des } \\
\text { partenaires } \\
\text { économiques } \\
\text { partenaires du marché } \\
\text { du travail (CPMT) } \\
\text { Conseil consultatif sur } \\
\text { le travail et la main- } \\
\text { d'œuvre (CCTM) }\end{array}$ \\
\hline Sectoriel & - & $\begin{array}{c}\text { Comités sectoriels de } \\
\text { main-d'œuvre (CSMO) }\end{array}$ \\
\hline Régional & $\begin{array}{c}\text { Comités régionaux } \\
\text { des programmes } \\
\text { ACCORD }\end{array}$ & $\begin{array}{c}\text { Comités régionaux des } \\
\text { partenaires du marché } \\
\text { du travail (CRPMT) }\end{array}$ \\
\hline Local & $\begin{array}{c}\text { Centres locaux de } \\
\text { développement } \\
\text { (CLD) }\end{array}$ & \\
\hline \multicolumn{2}{|c|}{} \\
\hline
\end{tabular}

Au niveau sectoriel, les représentants patronaux et syndicaux, ainsi que les instances gouvernementales et les organismes en lien avec les secteurs visés, siègent au sein de trente Comités sectoriels de maind'œuvre (CSMO). Leur rôle est de définir les besoins en développement de la main-d'œuvre propres à leur secteur d'activité, de proposer des mesures pour stabiliser l'emploi ou réduire le chômage, et développer la formation continue.

Au niveau régional, le gouvernement du Québec a également créé dix-sept Comités régionaux des partenaires du marché du travail (CRPMT) qui ont pour mandat d'élaborer diverses solutions pour remédier aux problématiques spécifiques qui se posent sur leurs marchés du travail. Il a également mis en place le projet d'Action concertée de coopération régionale de développement (ACCORD) en 2002 afin de favoriser le développement économique régional. Les comités régionaux du projet ACCORD détiennent le pouvoir de signer, au nom de la région, des ententes pour la mise en œuvre de plans de développement de créneaux d'excellence (bio-industries, matériaux composites, microélectronique, transformation du bois, etc.), qui permettent à une région de se positionner sur des marchés sur la base d'avantages comparatifs qui lui sont propres. Ces comités sont constitués principalement d'acteurs locaux : industriels, représentants de diverses institutions et élus. Bien que leur présence ne soit pas formellement assurée, les organisations syndicales sont impliquées dans quelques-uns de ces comités.

$\mathrm{Au}$ niveau local, les représentants des milieux d'affaires participent également aux projets menés par les Centres locaux de développement (CLD), dont la gestion est confiée aux municipalités régionales de comtés (MRC), dont l'objectif est de mettre en œuvre une action concertée en matière de développement local et de services de proximité. Si leur présence n'est pas systématique, les syndicats sont néanmoins actifs au sein de certains CLD.

\section{La concertation sociale comme amortisseur de la crise}

Nos entretiens auprès des représentants des principales organisations syndicales et patronales du Québec ont fait ressortir le rôle central joué par la Commission des partenaires du marché du travail (CPMT) dans la gestion de la crise de 2008-2009. Les principales mesures de sauvegarde de l'emploi ont en effet été définies au sein de cette institution qui intervient au niveau provincial.

Durant la crise, les syndicats et les employeurs ont participé à l'élaboration d'un Pacte pour l'emploi plus afin de limiter les licenciements collectifs par des mesures de soutien à la gestion des ressources humaines (par exemple une aide individualisée a été proposée pour la gestion de la performance au travail et celle des mouvements de main-d'œuvre), à la formation professionnelle, à l'intégration des immigrants et des minorités visibles. Ce programme prévoyait également la constitution d'équipes d'intervention régionale (table ad hoc de concertation) afin d'aider les entreprises à élaborer un plan d'action à court terme leur permettant de surmonter le ralentissement économique sans licencier leurs personnels. Comme on peut le constater sur le graphique 1, le soutien à la formation professionnelle a été la mesure la plus utilisée par les entreprises du Québec au cours de la crise. Elles ont également eu davantage recours aux mesures de services conseils de gestion de l'entreprise et de gestion des ressources humaines. D'après les données de la Direction de la statistique du ministère de l'Emploi et de la Solidarité sociale, le nombre d'entreprises qui ont bénéficié de ces mesures est passé de 10269 à 13763 entre 2007 et $2010 ; 76 \%$ d'entre elles sont des petites et moyennes entreprises (PME, 49 employés et moins).

Dans le cadre de la CPMT, les organisations syndicales et patronales ont également élaboré et mis en application un programme de Soutien aux entreprises à risque de ralentissement économique (SERRÉ) qui subventionne les entreprises pour qu'elles puissent réduire leurs heures de travail afin de développer les compétences de leur personnel grâce à des programmes de formation professionnelle. Son financement est assuré par les cotisations obligatoires des entreprises à un fonds provincial de formation ${ }^{(10)}$. Entre 2008 et 2010, ce programme aura permis de mettre sur pied 1192 projets de formation, d'aider 1092 entreprises et près de 28000 travailleurs, ce qui représente un engagement

(10) Les entreprises qui ne consacrent pas l'équivalent de $1 \%$ de leur masse salariale à des activités de formation doivent cotiser à hauteur de ce taux au Fonds de développement et de reconnaissance des compétences de la main-d'œuvre. 
financier d'environ 68,5 millions de dollars canadiens. Il a aussi contribué à aider les entreprises qui n'avaient plus de liquidités à traverser la crise.

Graphique 1 : Nombre d'entreprises bénéficiaires des mesures du Pacte pour l'emploi plus, 2007-2010

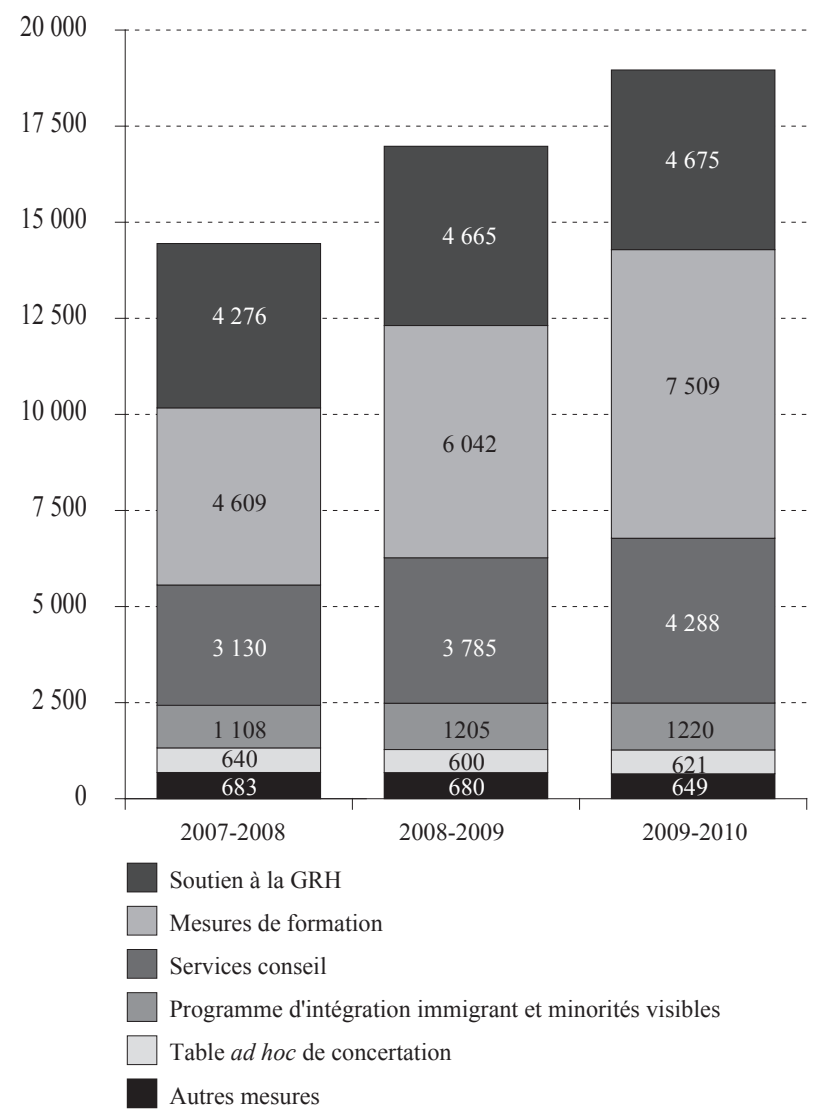

Source : Direction de la statistique du ministère de l'Emploi et de la Solidarité sociale.

Lorsqu'une entreprise a fait appel à l'une ou l'autre de ces mesures, elle a également été encouragée à se prévaloir du Programme de temps de travail partagé mis en place par le gouvernement fédéral canadien, qui a pour but d'éviter les licenciements collectifs lors des périodes de ralentissement de l'activité par une réduction des heures de travail. Les pertes de revenus des travailleurs concernés sont partiellement compensées par le versement d'allocations de chômage à même le fonds de l'assurance emploi. $\mathrm{Au}$ cours de la crise, le gouvernement a étendu la durée maximale durant laquelle une entreprise peut bénéficier de ce programme de 26 à 78 semaines. Il a aussi élargi son champ d'application en assouplissant ses critères d'admissibilité et en simplifiant les procédures de demandes pour les employeurs. Au Québec, le nombre de prestataires du programme de travail partagé est passé de 973 par mois en 2008, à 11266 en 2009, et à 14965 en 2010 (graphique 2).
Graphique 2 : Moyenne mensuelle du nombre de travailleurs bénéficiaires du programme fédéral de temps de travail partagé au Québec, en Ontario et au Canada, 2007-2010

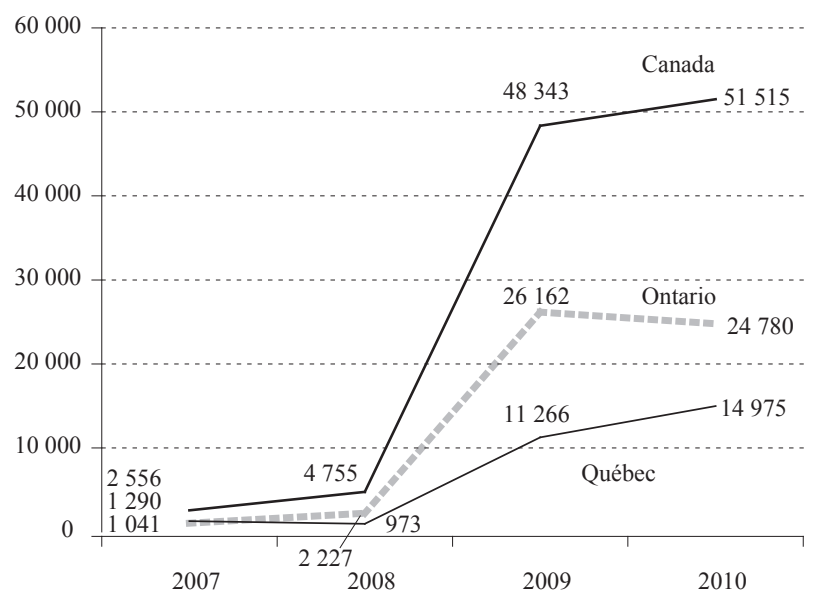

Source : Ressources humaines et développement des compétences Canada.

Il ne fait aucun doute pour les dirigeants des organisations syndicales et patronales que nous avons rencontrés que la mise en œuvre de ces mesures actives d'emploi a contribué à atténuer significativement les effets de la récession de 2008-2009 sur l'emploi. Comme l'indique le secrétaire général d'une des principales organisations syndicales du Québec :

«Lors de la crise économique de 1981-1982, on a découvert que la structure économique du Québec $n$ 'existait pas. On avait une industrie qui était vraiment gérée de l'extérieur du Québec. [...] Depuis ce momentlà, on a mis en place toute une série d'instruments de concertation qui sont assez uniques. On a appelé cela le "Québec Inc." [...] Ça nous permet de prendre des décisions sur ce que les employeurs et les travailleurs ont besoin sur le marché du travail. [...] En 2009, notre économie a résisté et ce n'est pas elle qui s'est ramassée en queue de peloton. Notre économie était diversifiée. On avait les instruments pour intervenir $\gg(11)$.

Le président d'une autre grande confédération syndicale confirme :

«J'ai vécu les crises des années 1980, du début des années 1990 et la dernière. Pour l'emploi, dans les trois crises que l'on a vécues, celle où l'on était le mieux outillé pour y faire face c'est la dernière. [...] Il n'y a pas beaucoup de pays dans le monde où l'on paye [les travailleurs] pour retourner sur les bancs d'écoles au lieu de les voir au chômage. [...] C'est au-delà de 1000 entreprises et de 25000 personnes [concernées]. [...] Il y avait [aussi] toute la question de la liquidité des entreprises qui n'avaient plus d'argent. [...] Et ça a fait en sorte que l'on n'a presque pas eu de faillites... [...] Tout cela est venu aussi de la Commission des partenaires économiques et de la CPMT. Il y a des structures sectorielles et régionales qui sont hyper efficaces ${ }^{(12)}$. 
Ce point de vue est partagé par le premier viceprésident d'une confédération patronale ${ }^{(13)} \mathrm{du}$ Québec : «Je pense que ces institutions [de concertation] ont été efficaces. [...] On siège sur plusieurs organismes paritaires [...] et ça marche. Ces structures ont encore une bonne espérance de vie parce que ça demeure pertinent $[. .]$.$» .$

Il convient en effet d'interpréter l'action menée par les acteurs syndicaux et patronaux au sein de ces institutions de concertation à la lumière des spécificités de la structure industrielle du Québec. Historiquement, celle-ci s'est développée sous l'impulsion des foyers d'industrialisation, des capitaux, des techniques et des compétences de gestion américains (FAUCHER, 1965). En d'autres termes, c'est à partir des États-Unis que s'est développée la structure industrielle du Québec, comme celle des autres provinces canadiennes, dans un contexte de forte dépendance aux capitaux et aux marchés provenant de ce pays. Les grandes firmes canadiennes qui se sont constituées dans ce cadre ont donc été étroitement contrôlées par les États-Unis. Cela explique, du moins en partie, que l'industrie canadienne se soit traditionnellement orientée vers l'exportation, majoritairement vers le marché américain, de matières premières, de minerais et de biens semifinis, et importe des États-Unis des produits finis, des équipements et des services techniques. Cette dépendance et ce contrôle de l'industrie québécoise et canadienne par les États-Unis se sont poursuivis durant tout le $\mathrm{Xx}^{\mathrm{e}}$ siècle et continuent, encore aujourd'hui, à structurer des pans importants de l'économie du Québec, en particulier dans certains secteurs de l'industrie manufacturière comme celui des pâtes et papiers (BARRÉ, Rioux, 2012).

Depuis la fin des années 1970, le Québec a entamé un vaste mouvement de modernisation technologique de ses entreprises et d'élargissement de leurs marchés. Un modèle de développement assez singulier à l'échelle de l'Amérique du Nord prend donc progressivement forme au Québec (BouRQuE, 2000), qui s'appuie sur le contrôle progressif des grandes entreprises privées par des administrateurs québécois, la création d'agences publiques autonomes (Investissement Québec, Caisse de dépôt et de placement du Québec, etc.) et d'entreprises publiques (Hydro-Québec par exemple). Il se caractérise par la mise en place d'une démarche partenariale avec la société civile et d'une concertation sociale avec les acteurs syndicaux et patronaux. Les institutions de concertation qui se sont développées au Québec durant les années 1980 et 1990 s'inscrivent ainsi dans un objectif de restructuration et de modernisation industrielle.

En 2008-2009, les acteurs collectifs du travail du Québec ont sans aucun doute réagi rapidement

(13) Entretien effectué le 20 avril 2010. à la crise économique et financière en se mobilisant dans le cadre des institutions de concertation. D'après les entretiens que nous avons effectués, il ressort assez clairement que les mesures temporaires qu'ils ont prises pour éviter les licenciements collectifs témoignent des capacités d'action offertes par le contexte institutionnel de concertation dans lequel ils évoluent. Ces mesures ont certainement permis de sauver un nombre important d'emplois, en particulier dans les secteurs des services et à forte intensité technologique plutôt que dans certaines industries où les trajectoires de restructuration privilégiées ont plutôt misé sur les délocalisations, le recours à la sous-traitance internationale et d'autres formes de restructurations industrielles conduisant à des pertes d'emplois définitives.

En somme, l'expérience québécoise montre une volonté affirmée des partenaires sociaux d'aménager de nouvelles formes d'institutions intermédiaires qui s'inscrivent dans la lignée du mouvement de responsabilisation des acteurs patronaux et syndicaux sur des enjeux divers tels la formation (BERNIER et al., 2003). Pour autant, plusieurs obstacles sont à surmonter par les acteurs.

L'obstacle principal est sans aucun doute d'ordre structurel et réside dans certaines particularités du système québécois de relations industrielles, régime décentralisé qui ne fait pas du niveau sectoriel le lieu privilégié de représentation et d'action des partenaires sociaux. Bien que la partie syndicale soit mieux structurée que la partie patronale au-delà des frontières de l'entreprise, il n'en demeure pas moins qu'elle éprouve des difficultés à s'engager au nom de l'ensemble des travailleurs d'un secteur lors des discussions de niveau sectoriel. En fait, le monopole de représentation de tous les salariés qui lui est accordé au niveau de l'établissement pose la question de sa représentativité au-delà de ses propres membres, surtout dans les secteurs faiblement syndiqués.

Une autre difficulté provient du fait que les expériences de concertation impliquent un changement radical dans la nature des rapports entre les acteurs. Processus volontaire et consensuel, la concertation doit être soutenue par une dynamique de coopération. Elle se distingue à la fois de l'activité de négociation collective et des rapports de concurrence qui caractérisent les relations d'affaires (Charest, 1998). Elle nécessite donc le passage difficile d'une dynamique de confrontation et de concurrence à une dynamique de coopération. De plus, il faut souligner l'absence de mécanismes de régulation des conflits en cas d'échec de la concertation dans un système qui repose sur la règle du consensus entre les acteurs, ce qui peut limiter les projets ou les innovations institutionnelles.

Ces modes de régulation inspireraient également des craintes aux acteurs, en particulier aux organisations syndicales. La concertation poursuivrait essentiellement un objectif d'amélioration de 
l'efficacité économique d'un secteur plus que celui de permettre à tous d'exprimer leurs véritables besoins, notamment ceux relatifs à la sécurité des travailleurs. Il y aurait donc un risque que les représentants des travailleurs soient réduits à de simples courroies de transmission des objectifs du patronat et du gouvernement (Rouillard, 2009). De plus, s'il existe un droit de la négociation collective qui assure l'équilibre des pouvoirs entre les parties et une qualification juridique aux accords négociés, ce n'est certainement pas le cas du dialogue social : la contrainte est en effet généralement absente de la démarche de dialogue social (JOBERT, 2008; BEVORT, JOBERT, 2011) qui repose plutôt sur un processus non contraignant résultant de la diffusion d'orientations de contenus et d'instruments de politiques publiques (Hassenteufel, 2005).

Dès lors, il faut bien comprendre que le modèle de concertation sociale repose sur la volonté des acteurs sociaux de le maintenir, voire de le renforcer. Les règles établies dans les différents espaces de discussion dont nous avons parlé doivent ensuite être diffusées afin de devenir une norme légitime appliquée par bon nombre d'acteurs appelés à jouer des rôles actifs à des niveaux inférieurs du système de relations industrielles. En d'autres termes, l'efficacité de la concertation est en partie tributaire de la capacité des partenaires sociaux à élaborer des lignes directrices qui serviront de guides pour les acteurs agissant au plan local. Ces constats montrent l'importance d'établir des connexions entre les différents niveaux de régulation du système de relations industrielles (MOREaU, 2006; Jobert, 2008). Bien plus, ils soulèvent la nécessité de comprendre l'articulation complexe entre les actions stratégiques qui se déploient au niveau de l'entreprise dans un dialogue bipartite institutionnalisé et le dialogue social qui s'opère à des niveaux supérieurs.

\section{Le découplage entre les niveaux de concertation et la négociation collective locale}

Les dirigeants syndicaux et patronaux que nous avons interrogés reconnaissent largement que la concertation sociale s'est renforcée lors de cette crise économique et financière. Ils sont cependant d'avis qu'il existe des obstacles majeurs à son efficacité en particulier parce que le couplage entre négociation décentralisée et négociation aux niveaux supérieurs de concertation ne se fait pas. Un représentant d'une confédération syndicale explique :

«Il y a des discussions, des consensus qui sortent de là [des institutions de concertation], mais ça a de la difficulté à atterrir sur le terrain. Quand vient le temps de concrétiser leurs décisions sur les lieux de travail, ça ne marche plus. [...] Du côté syndical on a de la difficulté à faire le lien entre cette concertation et la négociation. Comme il n'y a rien au niveau de la négociation sectorielle, ça se perd dans la réalité des négociations locales. On est satisfait du résultat des discussions mais ça n'a pas de répercussion concrète. On est bon pour identifier les problèmes (reconnaissance de compétences, problèmes de recrutement, etc.) et même pour trouver des solutions mais ça en reste là... »(14).

Le pouvoir attribué aux partenaires sociaux dans le cadre des institutions de concertation se limite en effet à un rôle strictement consultatif. À la différence de ce qui existe dans certains pays européens, ces institutions n'ont pas de pouvoir décisionnel au Québec et ne sont pas liées à des mécanismes, formels ou informels, qui permettraient de traduire les décisions qui en émanent dans des accords négociés entre syndicats et employeurs. De manière générale, les résultats de notre analyse des conventions collectives montrent que la volonté des acteurs sociaux du Québec de mettre en place un modèle permettant l'élaboration conjointe de mesures pour améliorer la compétitivité des entreprises et les conditions de vie des travailleurs ne semble pas se refléter dans les conventions collectives négociées au niveau des entreprises. Alors que les partenaires sociaux ont donné la priorité au développement du dialogue social au sein des entreprises, à l'amélioration de la protection des travailleurs et à la garantie d'une répartition équitable et transparente des gains de productivité, les acteurs impliqués dans la négociation collective au plan local n'ont pas retenu ces lignes directrices, exception faite de la formation professionnelle.

L'évolution des conventions collectives au Québec semble par ailleurs confirmer la thèse selon laquelle les employeurs ont, au cours de ces dernières années, poursuivi une logique de flexibilité et d'efficacité, sans octroyer aux travailleurs de contreparties significatives sur la sécurité d'emploi ou le partage des gains de productivité (JALETTE, LAROCHE, 2010) mais en apportant les modifications qui leur semblaient nécessaires aux conventions collectives. À titre d'exemple, les employeurs ont obtenu des syndicats des assouplissements sur les règles de promotion : la proportion de conventions collectives dont l'ancienneté constitue le seul critère de promotion est passée de $15,5 \%$ en 1988-1991 à 2,9\% en 2006-2009 et celle où l'ancienneté n'est pas prise en compte, de $8,9 \%$ à $23,3 \%$. L'accent mis sur une flexibilité accrue s'observe également sur le plan salarial : la proportion de conventions collectives comportant des dispositions en lien avec la rémunération au rendement, même si elle demeure faible en 2006-2009 (3,7\%), a presque doublé par rapport à 1988-1991 (1,9\%).

(14) Entretien effectué le 8 avril 2010. 
Nos résultats permettent cependant de constater une évolution favorable du dialogue social au niveau de l'entreprise par la mise en place de comités conjoints (patronaux-syndicaux) ad hoc. $\mathrm{Au}$ cours des vingt dernières années, nous observons une augmentation marquée de la proportion des conventions collectives qui contiennent des dispositions prévoyant des comités liés, d'une part, à l'application des conventions collectives (de 6,9\% entre 1988 et 1991 à 56,2 \% entre 2006 et 2009), et, d'autre part, à la formation (de 9,8\% entre 1988 et 1991 à $24,3 \%$ entre 2006 et 2009). Les comités chargés d'aborder des questions sur l'organisation du travail sont mentionnés relativement fréquemment dans les conventions collectives (19,7\% d'entre elles prévoient des dispositions à cet effet).

Sans que l'on puisse établir de relation de cause à effet, on peut noter que l'accroissement du nombre de comités conjoints est concomitant de l'importante diminution des conflits collectifs de travail au cours des dernières années ${ }^{(15)}$, ce qui reflète sans aucun doute la préoccupation des acteurs patronaux et syndicaux de maintenir une paix industrielle relative qu'ils estiment souvent essentielle à l'amélioration de la productivité et de la compétitivité des entreprises.

Mais, nous l'avons dit, que ce soit en matière de protection des emplois ou de partage équitable et transparent des gains de productivité, les syndicats ont enregistré des pertes considérables. Nos données témoignent notamment de réductions importantes des garanties salariales. La proportion des conventions collectives contenant de telles dispositions est passée de $31,1 \%$ entre 1988-1991 à $18,2 \%$ en 2006-2009. Les mesures de partage de l'emploi (de $5,3 \%$ à $1,9 \%$ ) ou de préavis contre les licenciements collectifs (de $8,9 \%$ à $3,2 \%$ ) ont également diminué. Jacques RouILLARD (2009) soutient également que malgré l'augmentation du produit intérieur brut et de la productivité au cours des vingt-cinq dernières années au Québec, le pouvoir d'achat des travailleurs n'a pas connu cette même tendance à la hausse.

Le tableau 2 permet d'examiner l'évolution des augmentations générales des salaires dans les ententes négociées au cours de la décennie 2000 parallèlement à celle du taux d'augmentation de l'indice des prix à la consommation (IPC) au Québec. Le premier constat est que le pouvoir d'achat de l'ensemble des syndiqués québécois n'a que faiblement augmenté entre 2000 et 2009 (les prix ayant progressé à un rythme de 20,7\% contre 21,9\% pour les salaires). Remarquons que les salariés du secteur privé ont davantage profité des augmentations de salaire $(24,7 \%)$ que ceux du secteur public $(+20,1 \%)$. Les salaires réels y ont même connu des évolutions divergentes : $+3,4 \%$ dans le privé et - $0,7 \%$ dans le public (calcul des auteurs non détaillés ici). Deux tendances opposées expliquent de tels résultats : la croissance économique qu'ont connue plusieurs secteurs et les menaces de fermeture et de délocalisation qui ont tempéré les revendications salariales des syndiqués (JALETTE, LAROCHE, 2008).

Tableau 2 : Évolution de l'indice des prix à la consommation (\% variation annuelle) et des taux de salaires négociés au Québec, 2000-2009

\begin{tabular}{|c|c|c|c|c|}
\cline { 3 - 5 } \multicolumn{2}{c|}{} & \multicolumn{3}{c|}{$\begin{array}{c}\text { Augmentations de salaire des } \\
\text { employés syndiqués }\end{array}$} \\
\hline Années & IPC & Ensemble & Privé & Public \\
\hline 2000 & 2,5 & 2,6 & 2,2 & 2,8 \\
\hline 2001 & 2,3 & 2,9 & 2,9 & 2,8 \\
\hline 2002 & 2 & 2,6 & 2,6 & 2,6 \\
\hline 2003 & 2,5 & 2,4 & 2,5 & 2,2 \\
\hline 2004 & 2 & 1,3 & 1,9 & 0,8 \\
\hline 2005 & 2,3 & 1,5 & 2,5 & 0,8 \\
\hline 2006 & 1,7 & 2,4 & 2,7 & 2,1 \\
\hline 2007 & 1,6 & 2,3 & 2,5 & 2,2 \\
\hline 2008 & 2,1 & 2 & 2,6 & 2,2 \\
\hline 2009 & 0,6 & 2,1 & 2,7 & 2,3 \\
\hline Total & $\mathbf{2 0 , 7}$ & $\mathbf{2 1 , 9}$ & $\mathbf{2 4 , 7}$ & $\mathbf{2 0 , 1}$ \\
\hline $\mathbf{2 0 0 0 - 2 0 0 9}$ & & & & \\
\hline
\end{tabular}

Note : L'évolution sur l'ensemble de la période a été calculée par nos soins. Sources : Institut de la statistique du Québec, principaux indicateurs économiques conjoncturels; ministère du Travail, indice de croissance des taux de salaires négociés (diverses années)

Si des dispositions relatives à l'indexation des salaires avaient auparavant été introduites dans les conventions collectives pour protéger le pouvoir d'achat des travailleurs, il en va désormais autrement. Selon notre analyse des ententes négociées au Québec entre 2007 et 2010, moins d'une convention sur cinq comporte une telle disposition (16\%), souvent obtenue à une époque antérieure ${ }^{(16)}$. Seulement quatre nouvelles dispositions en la matière ont été négociées alors que quatorze retraits ont été effectués. De tels résultats tendent à confirmer que les travailleurs n'ont obtenu que peu de garanties sur le maintien de leur pouvoir d'achat.

Par ailleurs, les conditions qui permettraient aux syndicats et aux travailleurs de participer activement à l'élaboration d'un modèle de développement économique de manière plus démocratique ne semblent pas non plus réunies. Contrairement à la situation européenne, il appartient aux parties patronales et salariées de négocier des dispositions conventionnelles prévoyant la transmission d'informations économiques, financières ou stratégiques. Or, les entreprises du Québec se montrent
(15) Le nombre annuel moyen de conflits est passé de 143,2 pour la période 1966-1970 à 61 pour la période 2005-2009: Ministère du Travail, Les arrêts de travail au Québec. Bilan annuel, Québec, ministère du Travail, diverses années.
(16) Analyse du contenu des ententes négociées au Québec entre 2007 et 2010 effectuée par les auteurs. Direction de l'information sur le travail, Information statistique sur le travail. Les ententes négociées, diverses années. 
peu enclines à adopter des dispositions conventionnelles les obligeant à divulguer de l'information de nature économique (Roy, et al., 2007). D'après nos données, entre 2006 et 2009, seulement 5,6\% des 6267 conventions collectives signées prévoyaient des dispositions à cet effet.

Comme nous l'avons mentionné plus haut, seule la question de la formation professionnelle demeure un point sur lequel nous observons l'existence d'un véritable couplage entre les instances de concertation et la négociation décentralisée. Nous constatons ainsi une augmentation significative de la proportion des conventions collectives incluant des dispositions sur la formation, le reclassement et le développement des ressources humaines (de 38,9 \% pour la période 1988-1991 à 69,4\% pour la période 2006-2009). Ces résultats ne sont pas surprenants, dans la mesure où il s'agit de la principale thématique abordée dans les instances de concertation sectorielles, régionales et même provinciales. En résumé, à l'exception des dispositions sur la formation professionnelle, les conventions collectives négociées au niveau des établissements ne prévoient pas d'autres mesures qui permettraient de gérer efficacement les chocs externes. L'amélioration de la protection conventionnelle relative à la formation professionnelle s'explique également, en grande partie, par les dispositions législatives adoptées au Québec dans le cadre de la loi du $1 \%{ }^{(17)}$. Cette dernière témoigne de l'importance qu'ont les mesures législatives dans la traduction des décisions prises par les partenaires sociaux au sein des institutions de concertation dans le contenu des accords qu'ils négocient au niveau des établissements. Par l'intermédiaire de la loi, les partenaires sociaux ont vu leurs efforts de concertation sociale en matière de formation professionnelle se traduire par une augmentation des dispositions conventionnelles traitant de cet objet.

Dans les autres domaines de concertation (protection des emplois, implication des travailleurs dans les décisions, répartition équitable des gains de productivité), les partenaires sociaux et le gouvernement devraient ainsi mener une réflexion collective sur la nécessité d'adopter des mesures législatives qui permettraient de transposer les priorités issues du dialogue sectoriel dans les dispositions négociées au niveau des établissements.

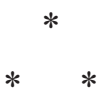

Cet article s'est interrogé sur les orientations prises par les acteurs sociaux du Québec dans la

(17) Loi favorisant le développement et la reconnaissance des compétences de la main-d'œuvre, du 8 juin 2007. Voir note $\mathrm{n}^{\circ} 10$. gestion de la crise économique et financière de 2008-2009. Comparativement à d'autres économies en Amérique du Nord et en Europe, cette province canadienne a connu des pertes d'emplois très limitées. Comme nous l'avons mis en évidence, une partie de l'explication de cette situation réside en la mutation profonde de l'économie québécoise en faveur des secteurs de services, compensant ainsi en partie les pertes massives d'emplois dans les industries manufacturières du Québec. Cette province n'a donc pas échappé aux effets de la crise sur l'emploi.

Nos observations nous amènent à conclure que les institutions de concertation sociale québécoises ont permis aux acteurs du monde du travail d'élaborer des solutions pour faire face à la crise et maintenir les niveaux d'emploi. Les mesures actives d'emploi qu'ils ont adoptées, ou adaptées à partir de programmes existants, ont de toute évidence aidé certaines entreprises à traverser la crise et limité fortement l'ampleur des licenciements collectifs. Elles n'ont cependant pas pu inverser le mouvement structurel de pertes massives d'emplois observable depuis le milieu des années 2000 dans certains secteurs industriels, en particulier dans le secteur manufacturier.

Le système hybride de relations collectives de travail qui caractérise le Québec, qui allie concertation centralisée et négociation décentralisée, reste marqué par une forte séparation de ces deux niveaux d'action. Les représentants qui siègent dans les différentes instances doivent non seulement représenter les intérêts de leurs membres, mais doivent également travailler conjointement avec les autres parties prenantes en recherchant l'intérêt commun. Ils doivent également servir de courroies de transmission auprès de leurs membres respectifs pour que les lignes directrices tracées au cours du processus de concertation soient réappropriées par les acteurs locaux et, par conséquent, appliquées à des niveaux inférieurs du système de relations industrielles. Nous reconnaissons que la démarche que nous avons adoptée pour étudier les liens potentiels entre les différents niveaux de régulation comporte certaines limites. Notre analyse s'est en effet uniquement concentrée sur les ententes négociées entre partenaires institutionnalisés. Elle nous a tout de même permis de rendre compte de l'état du dialogue social dans les entreprises syndiquées. Nous y avons observé une certaine évolution du dialogue social qui reflète la préoccupation des acteurs patronaux et syndicaux de maintenir une paix industrielle relative. La tradition de concertation observée aux niveaux sectoriel, régional ou même provincial s'est donc également développée au sein des entreprises.

En revanche, nos résultats montrent que les priorités et la vision des partenaires sociaux ne se traduisent pas dans le contenu des conventions collectives. Exception faite du développement des 
compétences de la main-d'œuvre, les données ne permettent pas de démontrer que la logique d'action locale s'inscrit directement dans la lignée des priorités définies par les grandes associations patronales et syndicales québécoises dans le cadre du dialogue social. Comment expliquer ce manque de connexion entre les niveaux de régulation? Nos entretiens ont mis en évidence que la vision que cultivent les partenaires sociaux dans les institutions de concertation se transpose difficilement au niveau de l'établissement. Dans un contexte de crise économique marqué par une très forte pression sur l'emploi, nos données indiquent que les acteurs qui agissent au niveau local semblent adopter des stratégies de nature défensive. Comme les ententes qu'ils négocient n'intègrent pas de dispositions conventionnelles permettant de mieux anticiper les chocs externes, il y a lieu de croire que, sans une transformation majeure de la nature des accords négociés, les représentants des employeurs et des travailleurs au niveau de l'entreprise demeureront tributaires des mesures d'urgence élaborées à des niveaux supérieurs de régulation.

Il ne fait aucun doute que les partenaires sociaux, qu'ils agissent au plan local ou à des niveaux supérieurs, seront appelés à très court terme à participer à une réflexion qui va les amener à reconsidérer les facteurs de compétitivité de leur économie. Or, à l'heure actuelle, les questions clés touchant au développement économique et social, notamment celles relatives à l'investissement technologique, l'amélioration de la productivité ou le rehaussement de la qualité et du niveau de vie des Québécois, échappent encore à tout espace de discussion entre les partenaires sociaux. Pourtant, si l'on peut tirer une leçon de la dernière crise économique, c'est bien que toutes les nations sont vulnérables aux soubresauts d'une économie mondialisée. Il y a donc fort à parier que ces questions demeureront d'actualité dans les prochaines années et pourraient stimuler le désir des partenaires sociaux de revoir les contours du modèle de concertation privilégié jusqu'à maintenant.

\section{Bibliographie}

Barré P., Rioux C. (2012), «L'industrie des produits forestiers au Québec : la crise d'un modèle socioproductif», Recherches sociographiques, vol. LIII, $\mathrm{n}^{\circ} 3$, pp. 645-669.

Bergeron J.-G., Bourque R. (1996), Worplace change in Quebec : public policy and union response, Kingston, IRC Press, Current Issues Series.

Bernard A. (2009), «Tendances de l'emploi manufacturier», Perspective, Statistique Canada, vol. 75, $\mathrm{n}^{\circ} 2$, pp. 5-14.

Bernier C., Frappier M., Moisan K. (2003), Repenser l'offre de formation publique pour le développement de la main-d'œuvre dans les PME du Québec, Québec, Centre multimédia CSQ, CSN.

Bevort A., Jobert A. (2011), Sociologie du travail : les relations professionnelles, Paris, Armand Colin, $2^{2}$ ed..

Bourque G.L. (2000), Le modèle québécois de développement. De l'émergence au renouvellement, Sainte-Foy, Presses de l'université du Québec.

ChARest J. (1998), Restructuration économique : transformations des relations industrielles et innovations institutionnelles : Configuration, émergence et impacts des initiatives sectorielles dans les contextes canadiens et québécois, Thèse de doctorat, École de relations industrielles, Université de Montréal.

Charest J. (2004), «The place of intermediate-level institutions in a decentralised industrial relations system : a Canadian perspective in the field of training $"$, in Cooney R., Stuart M. (eds.), Trade unions \& training : issues and international perspectives, Caulfield (Australia), National key centre in industrial relations, pp. 108-115.

Charest J. (2007), «Impacts of a law fostering training development : lessons from Quebec's experience», Administration publique du Canada - Canadian public administration, vol. 50, $\mathrm{n}^{\circ}$ 3, pp. 373-389.

Coutu M., Fontaine L.L., Marceau G. (2009), Droit des rapports collectifs de travail au Québec, Cowansville, Éditions Yvons Blais.

EUROFOUND (2009), Restructuring in Recession. ERM report 2009, European foundation for the improvement of living and working conditions, Office for official publications of the European communities, Luxembourg.

Eurofound (2010), Extending flexicurity. The potential of short-time working schemes. ERM report 2010, European foundation for the improvement of living and working conditions, Office for official publications of the European communities, Luxembourg.

Eurostat (2011), Enquête sur les forces de travail de l'Union européenne, Commission européenne, Office for official publications of the European communities, Luxembourg.

FAUCHER A. (1965), «Le caractère continental de l'industrialisation au Québec», Recherches sociographiques, vol. 6, n 3, pp. 219-236.

Freyssinet J. (2011), «L'impact de la crise économique sur les modes de production des normes de la relation d'emploi : l'exemple de six pays d'Europe occidentale», Économies et sociétés, Série «socio-économie du travail», n³3, pp. 1227-1264. 
Gagnon M.-J. (1991), «Le syndicalisme : du mode d'appréhension à l'objet sociologique», Sociologie et sociétés, vol. 23, n 2 , pp. 79-96.

Godin P. (1999), La révolution tranquille, 5 tomes, Montréal, Boréal.

Hassenteufel P. (2005), «De la comparaison internationale à la comparaison transnationale. Les déplacements de la construction d'objets comparatifs en matière de politiques publiques ", Revue française de science politique, vol. 55, $\mathrm{n}^{\circ}$ 1, pp. 113-132.

IsQ (2010), État du marché du travail au Québec. Bilan de l'année 2009, Institut de la statistique du Québec, Québec.

IsQ (2011), État du marché du travail au Québec. Bilan de l'année 2010, Institut de la statistique du Québec, Québec.

Jalette P., Laroche M. (2010), «Conclusion : tendances et orientations de la convention collective», in Jalette P., Trudeau G. (dir.), La convention collective au Québec, Montréal, Éditions Chenelière Éducation.

Jalette P., Prudent N. (2010), «Le secteur manufacturier la tête sous l'eau ", in Institut du nouveau monde, L'état du Québec en 2010, Montréal, Boréal, pp. 125-130.

Jobert A. (2008), Les nouveaux cadres du dialogue social, Europe et territoires, Bruxelles, PIE-Peter Lang.

LAROCHE M. (2007), L'impact de la mondialisation sur les structures, les stratégies et les institutions de négociation collective : le cas de la négociation coordonnée dans l'industrie du vêtement pour hommes au Québec, Thèse de doctorat, Gregor Murray (dir.), Département des relations industrielles, Université Laval, Québec.

Laroche M., Jalette P. (2008), «Où va le Québec en matière de relations du travail?», Revue Avantages, novembre, pp. 18-20.

Lefresne F., Sauviat C. (2011), «Les modèles sociaux européens et américains confrontés à la crise», Économies et sociétés, Série «socio-économie du travail», $\mathrm{n}^{\circ} 33$, pp. 1265-1300.

Ministère De L’emploi Et De La Solidarité Sociale (Mess) (2006), Pour une compétitivité accrue et un dialogue social renforcé. Partenaires pour la compétitivité et l'innovation sociale, Québec, Publications du Québec.

Ministère De L'industrie, Du Commerce Et De La Technologie (Mict) (1993), Un modèle d'entente de partenariat : le contrat social en entreprise, Québec, Publications du Québec.

Moreau M.-A. (2006), Normes sociales, droit du travail et mondialisation : confrontations et mutations, Paris, Éditions Dalloz.

Morin F. (1993), «La négociation collective selon le modèle de 1944 est-elle périmée?», in Bernier C. et al. (dir.), La négociation collective du travail : adaptation ou disparition?, Actes du XLIII ${ }^{\mathrm{e}}$ congrès des relations industrielles, Sainte-Foy, Presses de l'université Laval, pp. 13-44.

Niosi J. (1982), Les multinationales canadiennes, Montréal, Boréal Express.

Ocde (2011), Employment Outlook, Paris, OECD Publishing.

RouILlard J. (2009), L'expérience syndicale au Québec. Ses rapports à l'État, à la nation et à l'opinion publique, Montréal, VBL éditeur.

Roy M., Harrisson D., Haines V. (2007), «Le partenariat patronal-syndical et la gestion des conflits : les rôles clés des représentants », Gestion, vol. 31, n 4, pp. 9-15.

Uppal S. (2010), «La syndicalisation, $2010 »$, Perspective, Statistique Canada, vol. 11, $\mathrm{n}^{\circ} 10$

SpINEUx A. (dir.) (1999), «The institutional framework and the processes of collective bargaining within the 15 member states of the European Union», Dossier, $\mathrm{n}^{\circ} 17$, Université catholique de Louvain, Institut des sciences du travail. 